\title{
Long-term antibiotics for Chronic Rhinosinusitis: changing
}

\section{views}

Based on data from Asia showing the positive effect of longterm antibiotics in diffuse panbronchiolitis, a number of studies have been performed on the effect of long-term macrolides on symptomatology of chronic rhinosinusitis. Macrolides possess anti-inflammatory and immune-modulating effects and may be helpful in the treatment of CRS.

A few non-controlled studies have shown favourable results, especially in patients with recalcitrant CRS without nasal polyps (CRSsNP). These observations, combined with the impression in the clinic that patients' quality of life has indeed improved, called for placebo-controlled trials. Three relatively small double-blind placebo-controlled trials (total 176 patients) were performed and systematically reviewed which showed no effect after three months of macrolide treatment ${ }^{(1)}$. Furthermore, in recent years there has been considerable debate around the potential increased risks of cardiovascular events extending at least a year beyond exposure to the antibiotic ${ }^{(2)}$. In this issue of the Journal, Williamson et al. evaluated the risk of all-cause and cardiac death, and cardiovascular outcomes, associated with macrolide use in patients with CRS. They found, although not statistically significant, a potential increased short-term risk of myocardial infarction in patients with CRS following macrolide prescription. However, no evidence of longer-term increased risks was found.

Contrary to these unclear results in CRS, in the lower airways, and especially in COPD, a clear reduction of exacerbations has been found in patients with unstable disease ${ }^{(3)}$. The total number of patients evaluated, however, was much larger (3170 patients). One of the reasons of these differences in findings might be different pheno- and endotype of disease. In his first trial Wallwork has already highlighted potential differences in phenotype that might predict the effect of macrolides, with patients with low IgE having a better outcome, and recently a number of uncontrolled evaluations point to a potentially larger effect of long-term antibiotics in patients with non-Th2 forms of the disease ${ }^{(4,5)}$. The study in this Journal pointing at a shift towards eosinophilic inflammation, and away from nonTh2 forms of CRS in Asia in the last decade, may explain why, especially in Asia, macrolide therapy has been very successful in the past, although it could currently be expected to be less effective in that region ${ }^{(6)}$. A recent systematic review evaluated six prognostic factors to predict the favourable outcome of long-term macrolide therapy: CRS subtypes (CRSsNP versus CRSwNP), serum IgE level, membered lactone ring of macrolides, concurrent endoscopic sinus surgery (ESS), and dosage and duration of the macrolides. The authors concluded that CRSsNP and longer duration of treatment ( 24 weeks) were predictors of success ${ }^{(7)}$. The other factors were not helpful, particularly in the postoperative period, where there does not seem to be an additive effect of macrolides over nasal corticosteroids alone ${ }^{(7,8)}$. Recently it was proposed to systematically use eosinophils (as a marker of Th2 disease) on top of phenotyping based on the presence of nasal polyps, to differentiate treatments in CRS ${ }^{(9)}$. In EPOS2012, we decided to present long-term macrolides as an option although the evidence was thin. The upcoming EPOS2020 will again give a full systematic review of the relevant literature to help you to decide on the best treatment for your patients. For now, it is a close call to determine whether to prescribe macrolides and to whom.

\section{References}

1. Head K, Chong LY, Piromchai P, et al. Systemic and topical antibiotics for chronic rhinosinusitis. Cochrane Database Syst Rev. 2016;4:Cd011994.

2. Wong AYS, Chan EW, Anand S, Worsley AJ, Wong ICK. Managing Cardiovascular Risk of Macrolides: Systematic Review and Meta-Analysis. Drug saf. 2017;40(8):663-77.

3. Herath SC, Poole P. Prophylactic antibiotic therapy for chronic obstructive pulmonary disease (COPD). Cochrane Database Syst Rev. (11):Cd009764.

4. Deng J, Chen F, Lai Y, et al. Lack of additional effects of long-term, low-dose clarithromycin combined treatment compared with topical steroids alone for chronic rhinosinusitis in China: a randomized, controlled trial. Int Forum Allergy Rhinol. 2018;8(1):8-14.
5. Oakley GM, Christensen JM, Sacks R, Earls P, Harvey RJ. Characteristics of macrolide responders in persistent post-surgical rhinosinusitis. Rhinology. 2018;56(2):111-7.

6. Wei B, Liu F, Zhang J, et al. Multivariate analysis of inflammatory endotypes in recurrent nasal polyposis in a Chinese population. Rhinology. 2018;56(3):216-26.

7. Seresirikachorn K, Suwanparin N, Srisunthornphanich $C$, et al. Factors of success of low-dose macrolides in chronic sinusitis: Systematic review and meta-analysis. Laryngoscope. 2019;129(7):1510-9.

8. Zeng $\mathrm{M}$, Wang $\mathrm{H}$, Liao $\mathrm{B}$, Wang $\mathrm{H}$, Long $\mathrm{XB}, \mathrm{Ma}$ J, et al. Comparison of efficacy of fluticasone propionate versus clarithromycin for postoperative treatment of different phenotypic chronic rhinosinusitis: a randomized controlled trial. Rhinology.
2019;57(2):101-9.

9. Fokkens WJ, Reitsma S. Proposal for an Algorithm on the management of Chronic Rhinosinusitis. Allergy. 2019 Mar 27. doi: 10.1111/all.13797. [Epub ahead of print].

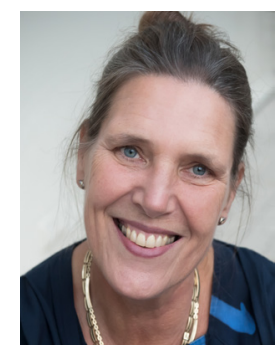

Wytske J. Fokkens, Editor-in Chief Amsterdam, the Netherlands 